\title{
ROTA-BAXTER 3-LIE ALGEBRAS
}

\author{
RUIPU BAI, LI GUO, JIAQIAN LI, AND YONG WU
}

\begin{abstract}
AвSTRACT. In this paper we introduce the concepts of a Rota-Baxter operator and a differential operator with weights on an $n$-algebra. We then focus on Rota-Baxter 3-Lie algebras and show that they can be derived from Rota-Baxter Lie algebras and pre-Lie algebras and from RotaBaxter commutative associative algebras with derivations. We also establish the inheritance property of Rota-Baxter 3-Lie algebras.
\end{abstract}

\section{INTRODUCTION}

$n$-Lie algebras [19] are a type of multiple algebraic systems appearing in many fields of mathematics and mathematical physics [33, 41, 4, 28, 27, 26, 34]. For example, the structure of 3-Lie algebras is applied to the study of the supersymmetry and gauge symmetry transformations of the world-volume theory of multiple coincident M2-branes; the Bagger-Lambert theory has a novel local gauge symmetry which is based on a metric 3-Lie algebra; the identity in Eq. (II) for a 3-Lie algebra is essential to define the action with $N=8$ supersymmetry; the $n$-Jacobi identity can be regarded as a generalized Plucker relation in the physics literature, and so on. The theory of $n$-Lie algebras has been widely studied [35, 36, 11], 12, 9, 10, 3].

P. Ho, Y. Imamura and Y. Matsuo in paper [29] studied two derivations of the multiple D2 action from the multiple M2-brane model proposed by Bagger-Lambert and Gustavsson. The first one is to start from 3-Lie algebras given by arbitrary Lie algebras through 2-dimensional extensions. The first author and collaborators [9] realized 3-Lie algebras by Lie algebras and several linear functions. Filippov [19] constructed $(n-1)$-Lie algebras from $n$-Lie algebras by fixing some non-zero vector in the multiplication. We are motivated by this to construct $n$-Lie algebras. However, it is not easy here due to the $n$-ary operation. In order to avoid the complicated equations involving the structural constants like in the classification [12], it is natural to consider to use Lie algebras and other better studied algebras to obtain 3-Lie and $n$-Lie algebras.

In recent years, Rota-Baxter (associative) algebras, originated from the work of G. Baxter [13] in probability and populated by the work of Cartier and Rota [14, 38, 39], have also been studied in connection with many areas of mathematics and physics, including combinatorics, number theory, operads and quantum field theory [1, 6, 17, 20, 21, 22, 24, 25, 38, 39]. In particular Rota-Baxter algebras have played an important role in the Hopf algebra approach of renormalization of perturbative quantum field theory of Connes and Kreimer [15, 17, 18], as well as in the application of the renormalization method in solving divergent problems in

Date: June 11, 2013.

2010 Mathematics Subject Classification. 17B05, 17D99.

Key words and phrases. Rota-Baxter operator, Rota-Baxter $n$-algebra, Rota-Baxter $n$-Lie algebra, differential $n$-algebra, pre-Lie algebra, Lie triple system. 
number theory [25, 32]. Furthermore, Rota-Baxter operators on a Lie algebra are an operator form of the classical Yang-Baxter equations and contribute to the study of integrable systems [5, 7, 8, 40]. Further Rota-Baxter Lie algebras are closely related to pre-Lie algebras and PostLie algebras.

Thus it is time to study $n$-Lie algebras and Rota-Baxter algebras together to get a suitable definition of Rota-Baxter $n$-Lie algebras. In this paper we investigate Rota-Baxter $n$-algebras in the context of associative and Lie algebras with focus on Rota-Baxter 3-Lie algebras. We establish a close relationship of our definition of Rota-Baxter 3-Lie algebras with well-known concepts of Rota-Baxter associative, commutative or Lie algebras. This on one hand justifies the definition of Rota-Baxter 3-Lie algebras and and on the other hand provides a rich source of examples for Rota-Baxter 3-Lie algebras. The concepts of differential operators and Rota-Baxter operators with weights for general (non-associative) algebras are introduced in Section 2 . The duality of the two concepts are established. In Section 3 we extend the connections [2, 9, 31] from Lie algebras and pre-Lie algebras to 3-Lie algebras to the context of Rota-Baxter 3-Lie algebras. In Section 4 , we construct Rota-Baxter 3-Lie algebras from commutative (associative) RotaBaxter algebras together with commuting derivations and suitable linear forms. In Section 5 we study the inheritance property of Rota-Baxter 3-Lie algebras as in the case of Rota-Baxter Lie algebras. We also consider the refined case of Lie triple systems.

\section{Differential $n$-Algebras and Rota-BaXter $n$-Lie algebras}

An $n$-Lie algebra is a vector space $g$ over a field $\mathbf{k}$ endowed with an $n$-ary multi-linear skewsymmetric operation $\left[x_{1}, \cdots, x_{n}\right]$ satisfying the $n$-Jacobi identity

$$
\left[\left[x_{1}, \cdots, x_{n}\right], y_{2}, \cdots, y_{n}\right]=\sum_{i=1}^{n}\left[x_{1}, \cdots,\left[x_{i}, y_{2}, \cdots, y_{n}\right], \cdots, x_{n}\right] .
$$

In particular, a 3-Lie algebra is a vector space $\mathfrak{g}$ endowed with a ternary multi-linear skewsymmetric operation

$$
\left[\left[x_{1}, x_{2}, x_{3}\right], y_{2}, y_{3}\right]=\left[\left[x_{1}, y_{2}, y_{3}\right], x_{2}, x_{3}\right]+\left[x_{1},\left[x_{2}, y_{2}, y_{3}\right], x_{3}\right]+\left[x_{1}, x_{2},\left[x_{3}, y_{2}, y_{3}\right]\right],
$$

for all $x_{1}, x_{2}, x_{3}, y_{2}, y_{3} \in \mathfrak{g}$. Under the skew-symmetric condition, the equation is equivalent to

$$
\left[\left[x_{1}, x_{2}, x_{3}\right], y_{2}, y_{3}\right]=\left[\left[x_{1}, y_{2}, y_{3}\right], x_{2}, x_{3}\right]+\left[\left[x_{2}, y_{2}, y_{3}\right], x_{3}, x_{1}\right]+\left[\left[x_{3}, y_{2}, y_{3}\right], x_{1}, x_{2}\right] \text {. }
$$

Let $(A, \cdot)$ be a k-vector space with a binary operation $\cdot$ and let $\lambda \in \mathbf{k}$. If a linear map $P: A \rightarrow A$ satisfies

$$
P(x) \cdot P(y)=P(P(x) \cdot y+x \cdot P(y)+\lambda x \cdot y) \text { for all } x, y \in A,
$$

then $P$ is called a Rota-Baxter operator of weight $\lambda$ and $(A, \cdot, P)$ is called a Rota-Baxter algebra of weight $\lambda$.

If a linear map $D: A \rightarrow A$ satisfies

$$
D(x \cdot y)=D(x) \cdot y+x \cdot D(y) \text { for all } x, y \in A,
$$

then $D$ is called a derivation on $A$. Let $\operatorname{Der}(A)$ denote the set of all derivations of $A$. More generally, a linear map $d: A \rightarrow A$ is called a derivation of weight $\lambda[23]$ if

$$
d(x y)=d(x) y+x d(y)+\lambda d(x) d(y), \quad \text { for all } x, y \in A .
$$

We generalize the concepts of a Rota-Baxter operator and differential operator to $n$-algebras. 
Definition 2.1. Let $\lambda \in k$ be fixed.

(a) An $n$-(nonassociative) algebra over a field $\mathbf{k}$ is a pair $(A,\langle, \cdots\rangle$,$) consisting of a vector$ space $A$ over $\mathbf{k}$ and a multilinear multiplication

$$
\langle, \cdots,\rangle: A^{\otimes n} \rightarrow A .
$$

(b) A derivation of weight $\lambda$ on an $n$-algebra $(A,\langle, \cdots\rangle$,$) is a linear map d: A \rightarrow A$ such that,

$$
d\left(\left\langle x_{1}, \cdots, x_{n}\right\rangle\right)=\sum_{\emptyset \neq I \subseteq[n]} \lambda^{|I|-1}\left\langle\breve{d}\left(x_{1}\right), \cdots, \breve{d}\left(x_{i}\right), \cdots, \breve{d}\left(x_{n}\right)\right\rangle
$$

where $\breve{d}\left(x_{i}\right):=\breve{d}_{I}\left(x_{i}\right):=\left\{\begin{array}{ll}d\left(x_{i}\right), & i \in I, \\ x_{i}, & i \notin I\end{array}\right.$ for all $x_{1}, \cdots, x_{n} \in A$. Then $A$ is called a differential $n$-algebra of weight $\lambda$. In particular, a differential 3-algebra of weight $\lambda$ is a 3-algebra $(A,\langle,\rangle$,$) with a linear map d: A \rightarrow A$ such that

$$
\begin{aligned}
d\left(\left\langle x_{1}, x_{2}, x_{3}\right\rangle\right)= & \left\langle d\left(x_{1}\right), x_{2}, x_{3}\right\rangle+\left\langle x_{1}, d\left(x_{2}\right), x_{3}\right\rangle+\left\langle x_{1}, x_{2}, d\left(x_{3}\right)\right\rangle \\
& +\lambda\left\langle d\left(x_{1}\right), d\left(x_{2}\right), x_{3}\right\rangle+\lambda\left\langle d\left(x_{1}\right), x_{2}, d\left(x_{3}\right)\right\rangle+\lambda\left\langle x_{1}, d\left(x_{2}\right), d\left(x_{3}\right)\right\rangle \\
& +\lambda^{2}\left\langle d\left(x_{1}\right), d\left(x_{2}\right), d\left(x_{3}\right)\right\rangle .
\end{aligned}
$$

(c) A Rota-Baxter operator of weight $\lambda$ on $(A,\langle, \cdots\rangle$,$) is a linear map P: A \rightarrow A$ such that

$$
\left\langle P\left(x_{1}\right), \cdots, P\left(x_{n}\right)\right\rangle=P\left(\sum_{\emptyset \neq I \subseteq[n]} \lambda^{|I|-1}\left\langle\hat{P}\left(x_{1}\right), \cdots, \hat{P}\left(x_{i}\right), \cdots, \hat{P}\left(x_{n}\right)\right\rangle\right),
$$

where $\hat{P}\left(x_{i}\right):=\hat{P}_{I}\left(x_{i}\right):=\left\{\begin{array}{ll}x_{i}, & i \in I, \\ P\left(x_{i}\right), & i \notin I\end{array}\right.$ for all $x_{1}, \cdots, x_{n} \in A$. Then $A$ is called a Rota-Baxter $n$-algebra of weight $\lambda$. In particular, a Rota-Baxter 3-algebra is a 3algebra $(A,\langle,\rangle$,$) with a linear map P: A \rightarrow A$ such that

$$
\begin{aligned}
\left\langle P\left(x_{1}\right), P\left(x_{2}\right), P\left(x_{3}\right)\right\rangle= & P\left(\left\langle P\left(x_{1}\right), P\left(x_{2}\right), x_{3}\right\rangle+\left\langle P\left(x_{1}\right), x_{2}, P\left(x_{3}\right)\right\rangle+\left\langle x_{1}, P\left(x_{2}\right), P\left(x_{3}\right)\right\rangle\right. \\
& +\lambda\left\langle P\left(x_{1}\right), x_{2}, x_{3}\right\rangle+\lambda\left\langle x_{1}, P\left(x_{2}\right), x_{3}\right\rangle+\lambda\left\langle x_{1}, x_{2}, P\left(x_{3}\right)\right\rangle \\
& \left.\left.+\lambda^{2}\left\langle x_{1}, x_{2}, x_{3}\right)\right\rangle\right) .
\end{aligned}
$$

Theorem 2.2. Let $(A,\langle, \cdots\rangle$,$) be an n-algebra over k$. An invertible linear mapping $P: A \rightarrow A$ is a Rota-Baxter operator of weight $\lambda$ on $A$ if and only if $P^{-1}$ is a differential operator of weight $\lambda$ on $A$.

Proof. If an invertible linear mapping $P: A \rightarrow A$ is a Rota-Baxter operator of weight $\lambda$, then for $x_{1}, \cdots, x_{n} \in A$, set $y_{i}=P^{-1}\left(x_{i}\right)$. Then by Eq. (9) we have

$$
\begin{aligned}
P^{-1}\left(\left\langle x_{1}, \cdots, x_{n}\right\rangle\right) & =P^{-1}\left(\left\langle P\left(y_{1}\right), \cdots, P\left(y_{n}\right)\right\rangle\right) \\
& =\sum_{\emptyset \neq I \subseteq[n]} \lambda^{|I|-1}\left\langle\hat{P}\left(y_{1}\right), \cdots, \hat{P}\left(y_{i}\right), \cdots, \hat{P}\left(y_{n}\right)\right\rangle \\
& =\sum_{\emptyset \neq I \subseteq[n]} \lambda^{|I|-1}\left\langle\hat{P} P^{-1}\left(x_{1}\right), \cdots, \hat{P} P^{-1}\left(x_{i}\right), \cdots, \hat{P} P^{-1}\left(x_{n}\right)\right\rangle
\end{aligned}
$$




$$
=\sum_{\emptyset \neq I \subseteq[n]} \lambda^{|I|-1}\left\langle\breve{P^{-1}}\left(x_{1}\right), \cdots, \breve{P^{-1}}\left(x_{i}\right), \cdots, \breve{P^{-1}}\left(x_{n}\right)\right\rangle .
$$

Therefore, $P^{-1}$ is a derivation of weight $\lambda$.

Conversely, let $P$ be a derivation of weight $\lambda$. For $x_{1}, \cdots, x_{n} \in A$, by Eq. (7) we have

$$
\begin{aligned}
P\left(\left\langle P^{-1}\left(x_{1}\right), \cdots, P^{-1}\left(x_{n}\right)\right\rangle\right) & =\sum_{\emptyset \neq I \subseteq[n]} \lambda^{|I|-1}\left\langle\check{P} P^{-1}\left(x_{1}\right), \cdots, \check{P} P^{-1}\left(x_{i}\right), \cdots, \check{P} P^{-1}\left(x_{n}\right)\right\rangle \\
& =\sum_{\emptyset \neq I \subseteq[n]} \lambda^{|I|-1}\left\langle\widehat{P^{-1}}\left(x_{1}\right), \cdots, \widehat{P^{-1}}\left(x_{i}\right), \cdots, \widehat{P^{-1}}\left(x_{n}\right)\right\rangle .
\end{aligned}
$$

Therefore,

$$
\left\langle P^{-1}\left(x_{1}\right), \cdots, P^{-1}\left(x_{n}\right)\right\rangle=P^{-1}\left(\sum_{\emptyset \neq I \subseteq[n]} \lambda^{|I|-1}\left\langle\widehat{P^{-1}}\left(x_{1}\right), \cdots, \widehat{P^{-1}}\left(x_{i}\right), \cdots, \widehat{P^{-1}}\left(x_{n}\right)\right\rangle\right) .
$$

This proves the result.

Proposition 2.3. Let $(A, \circ, P)$ be an associative Rota-Baxter (resp. differential) algebra of weight $\lambda$. Define an $n$-ary multiplication $\langle, \cdots$,$\rangle on A$ by

$$
\left\langle x_{1}, \cdots, x_{n}\right\rangle=x_{1} \circ \cdots \circ x_{n} \text { for all } x_{1}, \cdots, x_{n} \in A .
$$

Then $(A,\langle, \cdots\rangle, P$,$) is a Rota-Baxter (resp. differential) n-algebra of weight \lambda$.

Proof. We prove the case of Rota-Baxter algebras by induction on $n \geq 2$. The case of differential algebras is the same. There is nothing to prove when $n=2$. Suppose it is true for the case $n-1 \geq 2$. Then

$$
\begin{aligned}
& \left\langle P\left(x_{1}\right), \cdots, P\left(x_{n}\right)\right\rangle=\left(P\left(x_{1}\right) \circ \cdots \circ P\left(x_{n-1}\right)\right) \circ P\left(x_{n}\right) \\
& =\quad P\left(\sum_{I \subseteq[n-1]} \lambda^{|I|-1}\left\langle\hat{P}\left(x_{1}\right), \cdots, \hat{P}\left(x_{i}\right), \cdots, \hat{P}\left(x_{n-1}\right)\right\rangle\right) \circ P\left(x_{n}\right) \\
& =P\left\{P\left(\sum_{I \subseteq[n-1]} \lambda^{|I|-1}\left\langle\hat{P}\left(x_{1}\right), \cdots, \hat{P}\left(x_{i}\right), \cdots, \hat{P}\left(x_{n-1}\right)\right\rangle\right) \circ x_{n}\right. \\
& \quad+\left(\sum_{I \subseteq[n-1]} \lambda^{|I|-1}\left\langle\hat{P}\left(x_{1}\right), \cdots, \hat{P}\left(x_{i}\right), \cdots, \hat{P}\left(x_{n-1}\right)\right\rangle\right) \circ P\left(x_{n}\right) \\
& \left.\quad+\lambda\left(\sum_{I \subseteq[n-1]} \lambda^{|I|-1}\left\langle\hat{P}\left(x_{1}\right), \cdots, \hat{P}\left(x_{i}\right), \cdots, \hat{P}\left(x_{n-1}\right)\right\rangle\right) \circ x_{n}\right\} .
\end{aligned}
$$

The first sum inside $P$ gives $\left\langle P\left(x_{1}\right), \cdots, P\left(x_{n-1}\right), x_{n}\right\rangle$. Together with the third sum inside $P$, we obtain

The second sum inside $P$ is

$$
\sum_{\emptyset \neq I \subseteq[n], n \in I} \lambda^{|I|-1}\left\langle\hat{P}\left(x_{1}\right), \cdots, \hat{P}\left(x_{n}\right)\right\rangle .
$$

$$
\sum_{\emptyset \neq I \subseteq[n], n \notin I} \lambda^{|I|-1}\left\langle\hat{P}\left(x_{1}\right), \cdots, \hat{P}\left(x_{i}\right), \cdots, \hat{P}\left(x_{n}\right)\right\rangle .
$$

Therefore, $P$ is a Rota-Baxter operator of weight $\lambda$ on the $n$-algebra $(A,\langle, \cdots\rangle$,$) .$ 
3. Rota-Baxter 3-Lie algebras from Rota-Baxter LiE Algebras AND PRE-Lie ALGEBras

In this section, we study the realizations of Rota-Baxter 3-Lie algebras from Rota-Baxter Lie algebras in Section 3.1 and from Rota-Baxter pre-Lie algebras in Section 3.2.

3.1. Rota-Baxter 3-Lie algebras from Rota-Baxter Lie algebras. By Definition 2.1, suppose $(L,[,], P$,$) is a Rota-Baxter 3-Lie algebra of weight \lambda$. Then the linear map $P: L \rightarrow L$ satisfies

$$
\begin{aligned}
{\left[P\left(x_{1}\right), P\left(x_{2}\right), P\left(x_{3}\right)\right]=} & P\left(\left[P\left(x_{1}\right), P\left(x_{2}\right), x_{3}\right]+\left[P\left(x_{1}\right), x_{2}, P\left(x_{3}\right)\right]+\left[x_{1}, P\left(x_{2}\right), P\left(x_{3}\right)\right]\right. \\
& +\lambda\left[P\left(x_{1}\right), x_{2}, x_{3}\right]+\lambda\left[x_{1}, P\left(x_{2}\right), x_{3}\right]+\lambda\left[x_{1}, x_{2}, P\left(x_{3}\right)\right] \\
& \left.\left.+\lambda^{2}\left[x_{1}, x_{2}, x_{3}\right)\right]\right) \quad \text { for all } x_{1}, x_{2}, x_{3} \in L .
\end{aligned}
$$

We recall the following result from Lie algebras to 3-Lie algebras.

Lemma 3.1. [9] Let $(L,[]$,$) be a Lie algebra, and let f \in L^{*}:=\operatorname{Hom}(L, \mathbf{k})$ satisfy $f([x, y])=0$ for $x, y \in L$. Then $L$ is a 3-Lie algebra with the multiplication

$$
[x, y, z]_{f}:=f(x)[y, z]+f(y)[z, x]+f(z)[x, y] \text { for all } x, y, z \in L .
$$

We now combine this result with Rota-Baxter operators.

Theorem 3.2. Let $(L,[], P$,$) be a Rota-Baxter Lie algebra of weight \lambda, f \in L^{*}$ satisfying $f([x, y])=0$ for all $x, y \in L$. Then $P$ is a Rota-Baxter operator on the 3 -Lie algebra $\left(L,[,,]_{f}\right)$ define in $E q .(12)$ if and only if $P$ satisfies

$$
f(x)[P(y), P(z)]+f(y)[P(z), P(x)]+f(z)[P(x), P(y)] \in \operatorname{Ker}\left(P+\lambda I d_{L}\right) \text { for all } x, y, z \in L,
$$

where $I_{L}: L \rightarrow L$ is the identity map.

Proof. By Lemma 3.1, $L$ is a 3-Lie algebra with the multiplication [, , $]_{f}$ defined in Eq. (12). Now for arbitrary $x, y, z \in L$,

$$
\begin{aligned}
& {[P(x), P(y), P(z)]_{f}=f(P(x))[P(y), P(z)]+f(P(y))[P(z), P(x)]+f(P(z))[P(x), P(y)]} \\
& =\quad P(f(P(x)([P(y), z]+[y, P(z)])+f(P(y))([P(z), x]+[z, P(x)]) \\
& \quad+f(P(z))([P(x), y]+[x, P(y)])+\lambda(f(P(x))[y, z]+f(P(y))[z, x]+f(P(z))[x, y])) .
\end{aligned}
$$

Applying Eqs. (†), (12) and regrouping, we obtain

$$
\begin{aligned}
& P\left([P(x), P(y), z]_{f}+[P(x), y, P(z)]_{f}+[x, P(y), P(z)]_{f}\right. \\
& \left.+\lambda\left([P(x), y, z]_{f}+[x, P(y), z]_{f}+[x, y, P(z)]_{f}\right)+\lambda^{2}[x, y, z]_{f}\right) \\
= & P(f(P(x))([P(y), z]+[y, P(z)])+f(P(y))([z, P(x)]+[P(z), x]) \\
& +f(P(z))([P(x), y]+[x, P(y)])+\lambda(f(P(x))[y, z]+f(P(y))[z, x]+f(P(z))[x, y]) \\
& +\lambda(f(x)([P(y), z]+[z, P(y)]+\lambda[y, z])+f(y)([z, P(x)]+[P(z), x])+\lambda[z, x]) \\
& +f(z)([P(x), y]+[x, P(y)]+\lambda[x, y])+f(x)[P(y), P(z)]+f(y)[P(z), P(x)]+f(z)[P(x), P(y)]) \\
= & {[P(x), P(y), P(z)]_{f}+\left(P+\lambda I d_{L}\right)(f(x)[P(y), P(z)]+f(y)[P(z), P(x)]+f(z)[P(x), P(y)]) . }
\end{aligned}
$$

Then the theorem follows. 
Corollary 3.3. Let $(L,[], P$,$) be a Rota-Baxter Lie algebra of weight zero, f \in L^{*}$ satisfy $f([x, y])=0$ for all $x, y \in L$. Then $P$ is a Rota-Baxter operator on the 3 -Lie algebra $\left(L,[,,]_{f}\right)$ if and only if $P$ satisfies

$$
[f(x) P(y)-f(y) P(x), z]+[f(y) P(z)-f(z) P(y), x]+[f(z) P(x)-f(x) P(z), y] \in \operatorname{Ker} P^{2}
$$

for $x, y, z \in L$, where, $[,,]_{f}$ is defined in Eq.(12).

In particular, if $P^{2}=0$, then for every $f \in L^{*}$ satisfying $f([x, y])=0, P$ is a Rota-Baxter operator on the 3-Lie algebra $\left(L,[,,]_{f}\right)$.

Proof. Let $(L,[], P$,$) be a Rota-Baxter Lie algebra of weight zero, f \in L^{*}$ satisfying $f([x, y])=0$ for $x, y \in L$. By Eq. (\#) we have

$$
\begin{aligned}
& f(x)[P(y), P(z)]+f(y)[P(z), P(x)]+f(z)[P(x), P(y)] \\
= & P^{2}([f(x) P(y)-f(y) P(x), z]+[f(y) P(z)-f(z) P(y), x]+[f(z) P(x)-f(x) P(z), y]) .
\end{aligned}
$$

Then the corollary follows from Theorem 3.2 .

3.2. Rota-Baxter 3-Lie algebras from Rota-Baxter pre-Lie algebras. In this section, we study the realizations of Rota-Baxter 3-Lie algebras by Rota-Baxter associative algebras and Rota-Baxter pre-Lie algebras. First we recall some properties (cf. [31]).

Let $L$ be a vector space over a field $F$ with a bilinear product $*$ satisfying

$$
(x * y) * z-x *(y * z)=(y * x) * z-y *(x * z) \text { for all } x, y, z \in L .
$$

Then $(L, *)$ is called a pre-Lie algebra. It is obvious that all associative algebras are pre-Lie algebras. For a pre-Lie algebra $L$, the commutator

$$
[x, y]_{*}:=x * y-y * x,
$$

defines a Lie algebra $G(L)=\left(L,[.]_{*}\right)$, called the sub-adjacent Lie algebra of the pre-Lie algebra $L$.

If a linear mapping $P: L \rightarrow L$ is a Rota-Baxter operator of weight $\lambda$ on a pre-Lie algebra $(L, *)$, that is, $P$ satisfies

$$
P(x) * P(y)=P(P(x) * y+x * P(y)+\lambda x * y) \text { for all } x, y \in L,
$$

then $P$ is a Rota-Baxter operator of weight $\lambda$ on its sub-adjacent Lie algebra $G(L)=\left(L,[,]_{*}\right)$.

The following facts on relationship among various Rota-Baxter algebras can be easily verified from definitions.

Lemma 3.4. (a) Let $(L, *, P)$ be a Rota-Baxter pre-Lie algebra of weight $\lambda$. Then $\left(L,[,]_{*}, P\right)$ is a Rota-Baxter Lie algebra of weight $\lambda$, where the multiplication [, $]_{*}$ is defined in Eq. (16)

(b) If $(L, *, P)$ is a Rota-Baxter pre-Lie algebra of weight $\lambda$. Then $(L, \cdot, P)$ is a Rota-Baxter pre-Lie algebra of weight $\lambda$, where

$$
x \cdot y:=P(x) * y-y * P(x)+\lambda x * y \quad \text { for all } x, y \in L .
$$


(c) Let $(A, \cdot, P)$ be a Rota-Baxter commutative associative algebra of weight $\lambda, D$ be a derivation on the algebra $(A, \cdot)$ that satisfies $D P=P D$. Then $(A, *, P)$ is a Rota-Baxter pre-Lie algebra of weight $\lambda$, where

$$
x * y=D(x) \cdot y \quad \text { for all } x, y \in A .
$$

Therefore by Item $\left[(b)\right.$, we get a Rota-Baxter Lie algebra $\left(A,[,]_{*}\right)$, where

$$
[x, y]_{*}=D(x) \cdot y-D(y) \cdot x \quad \text { for all } x, y \in A .
$$

Theorem 3.5. Let $(L, *, P)$ be a Rota-Baxter pre-Lie algebra of weight $\lambda$ and let $f \in L^{*}$ satisfying $f(x * y-y * x)=0$ for $x, y \in L$. Define

(20) $[x, y, z]_{f}=f(x)(y * z-z * y)+f(y)(z * x-x * z)+f(z)(x * y-y * x)$ for all $x, y, z \in L$.

Then $P$ is a Rota-Baxter operator on 3-Lie algebra $\left(L,[,,]_{f}\right)$ if and only if

$$
\begin{aligned}
& f(x)(P(y) * P(z)-P(z) * P(y))+f(y)(P(z) * P(x)-P(x) * P(z)) \\
& \quad+f(z)(P(x) * P(y)-P(y) * P(x)) \in \operatorname{Ker}\left(P+\lambda I d_{L}\right) \text { for all } x, y, z \in L .
\end{aligned}
$$

Proof. By Lemma 3.1, for $f \in L^{*}$ satisfying $f(x * y-y * x)=0,\left(L,[,,]_{f}\right)$ is a 3-Lie algebra with the multiplication in Eq. (20). By Lemma 3.4. a) and Theorem 3.2, $P$ is a Rota-Baxter operator on the 3-Lie algebra $\left(L,[,,]_{f}\right)$ if and only if $P$ satisfies Eq. (21).

Theorem 3.6. Let $(L, *, P)$ be a Rota-Baxter pre-Lie algebra of weight zero, $f \in L^{*}$ satisfy

$$
f(P(x) * y-y * P(x))=f(P(y) * x-x * P(y)) \quad \text { for all } x, y \in L .
$$

Then $(L,[,]$,$) is a 3-Lie algebra with the multiplication$

$$
\begin{aligned}
{[x, y, z]:=} & (f(x) P(y)-f(y) P(x)) * z-z *(f(x) P(y)-f(y) P(x)) \\
& +(f(z) P(x)-f(x) P(z)) * y-y *(f(z) P(x)-f(x) P(z)) \\
& +(f(y) P(z)-f(z) P(y)) * x-x *(f(y) P(z)-f(z) P(y)) .
\end{aligned}
$$

Further, $P$ is a Rota-Baxter operator of weight zero on the 3 -Lie algebra $(L,[,]$,$) if and only if$ $P$ satisfies

$$
\begin{aligned}
& f(x)\left(P^{2}(y) * P^{2}(z)-P^{2}(z) * P^{2}(y)\right)+f(y)\left(P^{2}(z) * P^{2}(x)-P^{2}(x) * P^{2}(z)\right) \\
& +f(z)\left(P^{2}(x) * P^{2}(y)-P^{2}(y) * P^{2}(x)\right)=0 \quad \text { for all } x, y, z \in L .
\end{aligned}
$$

Proof. By Lemma 3.4.(b), $(L, \circ)$ is a pre-Lie algebra with the multiplication

$$
\circ: L \otimes L \rightarrow L, x \circ y=P(x) * y-y * P(x) \text { for all } x, y \in L,
$$

and $P$ is a Rota-Baxter operator on the pre-Lie algebra $(L, \circ)$.

If $f \in L^{*}$ satisfies $f(x \circ y-y \circ x)=0$, that is, $f$ satisfies Eq. (22), then by Lemma 3.1, $(L,[,]$, is a 3 -Lie algebra, where

$$
\begin{aligned}
{[x, y, z]=} & f(x)(y \circ z-z \circ y)+f(y)(z \circ x-x \circ z)+f(z)(x \circ y-y \circ x) \\
= & f(x)(P(y) * z-z * P(y)-P(z) * y+y * P(z)) \\
& +f(y)(P(z) * x-x * P(z)-P(x) * z+z * P(x)) \\
& +f(z)(P(x) * y-y * P(x)-P(y) * x+x * P(y)) \\
= & (f(x) P(y)-f(y) P(x)) * z-z *(f(x) P(y)-f(y) P(x))
\end{aligned}
$$




$$
\begin{aligned}
& +(f(z) P(x)-f(x) P(z)) * y-y *(f(z) P(x)-f(x) P(z)) \\
& +(f(y) P(z)-f(z) P(y)) * x-x *(f(y) P(z)-f(z) P(y)) .
\end{aligned}
$$

Therefore, Eq. (23) holds.

By Theorem 3.5, $P$ is a Rota-Baxter operator on the 3-Lie algebra $(L,[,]$,$) if and only if P$ satisfies

$$
\begin{aligned}
0= & P(f(x)(P(y) \circ P(z)-P(z) \circ P(y))+f(y)(P(z) \circ P(x)-P(x) \circ P(z)) \\
& +f(z)(P(x) \circ P(y)-P(y) \circ P(x))) \\
= & f(x) P\left(P^{2}(y) * P(z)-P(z) * P^{2}(y)-P^{2}(z) * P(y)+P(y) * P^{2}(z)\right) \\
& +f(y) P\left(P^{2}(z) * P(x)-P(x) * P^{2}(z)-P^{2}(x) * P(z)+P(z) * P^{2}(x)\right) \\
& +f(z) P\left(P^{2}(x) * P(y)-P(y) * P^{2}(x)-P^{2}(y) * P(x)+P(x) * P^{2}(y)\right) \\
= & f(x)\left(P^{2}(y) * P^{2}(z)-P^{2}(z) * P^{2}(y)+f(y)\left(P^{2}(z) * P^{2}(x)-P^{2}(x) * P^{2}(z)\right)\right. \\
& +f(z)\left(P^{2}(x) * P^{2}(y)-P^{2}(y) * P^{2}(x) .\right.
\end{aligned}
$$

This proves the second statement.

4. Rota-BaXter 3-Lie algebras From Rota-Baxter commutative ASSOciative algebras

Let $(A, \cdot)$ be a commutative associative algebra, $D$ in $\operatorname{Der} A, f$ in $A^{*}$ satisfying

$$
f(D(x) y)=f(x D(y)) \text {. }
$$

Then by Lemma 3.1 and Lemma 3.4.(c), $\left(A,[,,]_{f, D}\right)$ is a 3-Lie algebra, where

$$
\begin{aligned}
& {[x, y, z]_{f, D}:=\left|\begin{array}{ccc}
f(x) & f(y) & f(z) \\
D(x) & D(y) & D(z) \\
x & y & z
\end{array}\right|} \\
& \quad=f(x)(D(y) \cdot z-D(z) \cdot y)+f(y)(D(z) \cdot x-D(x) \cdot z)+f(z)(D(x) \cdot y-D(y) \cdot x) \\
& \quad=D(f(x) y-f(y) x) \cdot z+D(f(z) x-f(x) z) \cdot y+D(f(y) z-f(z) y) \cdot x
\end{aligned}
$$

for $x, y, z \in A$.

Theorem 4.1. Let $(A, \cdot, P)$ be a commutative associative Rota-Baxter algebra of weight $\lambda, D \in$ DerA satisfying $P D=D P$ and $f \in A^{*}$ satisfying $f(D(x) y)=f(x D(y))$. Then $P$ is a Rota-Baxter operator of weight $\lambda$ on the 3 -Lie algebra $\left(A,[,,]_{f, D}\right)$ if and only if $P$ satisfies

$$
\left|\begin{array}{ccc}
f(x) & f(y) & f(z) \\
D P(x) & D P(y) & D P(z) \\
P(x) & P(y) & P(z)
\end{array}\right| \in \operatorname{Ker}\left(P+\lambda I d_{L}\right), \text { for all } x, y, z \in A .
$$

Proof. The result follows directly from Theorem 3.2 and Lemma 3.4.(c).

We prove a lemma before giving our next results on Rota-Baxter 3-Lie algebras.

Lemma 4.2. Let $A$ be a commutative algebra. For a $3 \times 3$-matrix $M$, we use the notation

$$
M:=\left[\begin{array}{lll}
x_{1} & y_{1} & z_{1} \\
x_{2} & y_{2} & z_{2} \\
x_{3} & y_{3} & z_{3}
\end{array}\right]=\left[\begin{array}{lll}
\vec{x} & \vec{y} & \vec{z}
\end{array}\right]
$$


and the corresponding determinant, where $\vec{x}, \vec{y}$ and $\vec{z}$ denote the column vectors. Let $P: A \rightarrow A$ be a Rota-Baxter operator of weight $\lambda$ and let $P(\vec{x}), P(\vec{y})$ and $P(\vec{z})$ denote the images of the column vectors. Then we have

$$
|P(\vec{x}) \quad P(\vec{y}) \quad P(\vec{z})|=P\left(\sum_{\emptyset \neq I \subseteq[3]} \lambda^{|I|-1}|\hat{P}(\vec{x}) \quad \hat{P}(\vec{y}) \quad \hat{P}(\vec{z})|\right) .
$$

Proof. By the definition of determinants and Proposition 2.3, we have

$$
\begin{aligned}
& |P(\vec{x}) \quad P(\vec{y}) \quad P(\vec{z})|=\sum_{\sigma \in S_{3}} \operatorname{sgn}(\sigma) P\left(x_{\sigma(1)}\right) P\left(y_{\sigma(2)}\right) P\left(z_{\sigma(3)}\right) \\
& =\sum_{\sigma \in S_{3}} \operatorname{sgn}(\sigma) P\left(\sum_{\emptyset \neq I \subseteq[3]} \lambda^{|I|-1} \hat{P}\left(x_{\sigma(1)}\right) \hat{P}\left(y_{\sigma(2)}\right) \hat{P}\left(z_{\sigma(3)}\right)\right) \\
& =P\left(\sum_{\emptyset \neq I \subseteq[3]} \lambda^{|I|-1}|\hat{P}(\vec{x}) \quad \hat{P}(\vec{y}) \quad \hat{P}(\vec{z})|\right) \text {, }
\end{aligned}
$$

as needed.

Let $A$ be a commutative associative algebra, $D_{1}, D_{2}, D_{3}$ be derivations on $(A, \cdot)$ satisfying $D_{1} D_{2}=D_{2} D_{1}$ for $i, j=1,2$. Then by [16] $A$ is a 3-Lie algebra with the multiplication (28)

$$
\left[x_{1}, x_{2}, x_{3}\right]:=\left|\begin{array}{ccc}
x_{1} & x_{2} & x_{3} \\
D_{1}\left(x_{1}\right) & D_{1}\left(x_{2}\right) & D_{1}\left(x_{3}\right) \\
D_{2}\left(x_{1}\right) & D_{2}\left(x_{2}\right) & D_{2}\left(x_{3}\right)
\end{array}\right|=\left|\begin{array}{lll}
\vec{x} & D_{1}(\vec{x}) & D_{2}(\vec{x})
\end{array}\right| \text { for all } \vec{x}:=\left[x_{1}, x_{2}, x_{3}\right]^{T} \in A^{3} .
$$

Theorem 4.3. Let $(A, P)$ be a Rota-Baxter commutative associative algebra of weight $\lambda, D_{1}, D_{2}$ be derivations on $(A, \cdot)$ satisfying $D_{1} D_{2}=D_{2} D_{1}, P D_{i}=D_{i} P$ for $i=1,2$. Then $P$ is a RotaBaxter operator of weight $\lambda$ on the 3-Lie algebra $(A,[,]$,$) , where [,$,$] is defined by Eq. (28).$

Proof. Let $x_{1}, x_{2}, x_{3} \in A$. Since $P D_{1}=D_{1} P$ and $P D_{2}=D_{2} P$, by Lemma 4.2 and Eq. (28) we have

$$
\begin{aligned}
& {\left[P\left(x_{1}\right), P\left(x_{2}\right), P\left(x_{3}\right)\right]=\left|\begin{array}{lll}
P(\vec{x}) & D_{1}(P(\vec{x})) & D_{2}(P(\vec{x}))
\end{array}\right|} \\
& =\left|\begin{array}{lll}
P(\vec{x}) & P\left(D_{1}(\vec{x})\right) & P\left(D_{2}(\vec{x})\right)
\end{array}\right| \\
& =P\left(\sum_{\emptyset \neq I \subseteq[3]} \lambda^{|I|-1}\left|\hat{P}(\vec{x}) \hat{P}\left(D_{1}(\vec{x})\right) \quad \hat{P}\left(D_{2}(\vec{x})\right)\right|\right) \\
& =P\left(\sum_{\emptyset \neq I \subseteq[3]} \lambda^{|I|-1}\left|\hat{P}(\vec{x}) \quad D_{1}(\hat{P}(\vec{x})) \quad D_{2}(\hat{P}(\vec{x}))\right|\right) \\
& =P\left(\sum_{\emptyset \neq I \subseteq[3]} \lambda^{|I|-1}\left[\hat{P}\left(x_{1}\right), \hat{P}\left(x_{2}\right), \hat{P}\left(x_{3}\right)\right]\right) \text {. }
\end{aligned}
$$

This is what we need. 
Let $A$ be a commutative associative algebra, $D_{1}, D_{2}, D_{3}$ be derivations on $A$ satisfying $D_{i} D_{j}=$ $D_{j} D_{i}$ for $i \neq j, i, j=1,2,3$. Then by [19] $A$ is a 3-Lie algebra with the multiplication (29)

$$
[x, y, z]_{D}:=\left|\begin{array}{lll}
D_{1}\left(x_{1}\right) & D_{1}\left(x_{2}\right) & D_{1}\left(x_{3}\right) \\
D_{2}\left(x_{1}\right) & D_{2}\left(x_{2}\right) & D_{2}\left(x_{3}\right) \\
D_{3}\left(x_{1}\right) & D_{3}\left(x_{2}\right) & D_{3}\left(x_{3}\right)
\end{array}\right|=\left|\begin{array}{lll}
D_{1}(\vec{x}) & D_{2}(\vec{x}) & D_{3}(\vec{x})
\end{array}\right| \text {, for all } \vec{x}=\left[x_{1}, x_{2}, x_{3}\right]^{T} \in A^{3} .
$$

Theorem 4.4. Let $(A, P)$ be a Rota-Baxter commutative associative algebra of weight $\lambda, D_{1}, D_{2}, D_{3}$ be derivations of $(A, \cdot)$ satisfying $D_{i} D_{j}=D_{j} D_{i}$ and $P D_{i}=D_{i} P$ for $i, j=1,2,3, i \neq j$. Then $P$ is a Rota-Baxter operator of weight $\lambda$ on the 3 -Lie algebra $\left(A,[,,]_{D}\right)$, where the multiplication $[,,]_{D}$ is defined by Eq. (29).

Proof. The proof follows the same argument as the proof for Theorem t.3.

\section{INHERITANCE PROPERTIES OF Rota-BAXTER 3-Lie ALGEBRAS}

In this section, we study the inheritance properties of Rota-Baxter 3-Lie algebras. Such a property of Rota-Baxter Lie algebras plays an important role in their theoretical study and applications such as in integrable systems [5, 0, 40]. This is presented in Section 5.1. We also establish a similar property for Rota-Baxter Lie triple systems in Section 5.2.

5.1. Rota-Baxter 3-Lie algebras constructed by Rota-Baxter 3 -Lie algebras. Let $(L,[,], P$, be a Rota-Baxter 3-Lie algebra of weight $\lambda$. Using the notation in Eq. (9), we define a ternary operation on $L$ by

$$
\begin{aligned}
& {\left[x_{1}, x_{2}, x_{3}\right]_{P}=\sum_{\emptyset \neq I \subseteq[3]} \lambda^{|l|-1}\left[\hat{P}_{I}\left(x_{1}\right), \hat{P}_{I}\left(x_{2}\right), \hat{P}_{I}\left(x_{3}\right)\right] } \\
&= \quad[P(x), P(y), z]+[P(x), y, P(z)]+[x, P(y), P(z)] \\
&+\lambda[P(x), y, z]+\lambda[x, P(y), z]+\lambda[x, y, P(z)]+\lambda^{2}[x, y, z] \text { for all } x, y, z \in L .
\end{aligned}
$$

Then we have the following result.

Theorem 5.1. Let $(L,[,], P$,$) be a Rota-Baxter 3-Lie algebra of weight \lambda$. Then with $[,,$,$] in$ Eq. (30), $\left(L,[,,]_{P}, P\right)$ is a Rota-Baxter 3-Lie algebra of weight $\lambda$.

Proof. First we prove that $\left(L,[,]_{P}\right)$ is a 3 -Lie algebra. It is clear that $[,,]_{P}$ is multi-linear and skew-symmetric.

Let $x_{1}, x_{2}, x_{3}, x_{4}, x_{5} \in L$. Denote $y_{1}=\left[x_{1}, x_{2}, x_{3}\right]_{P}, y_{2}=x_{4}, y_{3}=x_{5}$. Then by Eqs. (1D), (19) and (30), we have

$$
\begin{aligned}
{\left[\left[x_{1}, x_{2}, x_{3}\right]_{P}, x_{4}, x_{5}\right]_{P} } & =\left[y_{1}, y_{2}, y_{3}\right]_{P} \\
& =\sum_{\emptyset \neq I \subseteq[3]} \lambda^{[I \mid-1}\left[\hat{P}_{I}\left(y_{1}\right), \hat{P}_{I}\left(y_{2}\right), \hat{P}_{I}\left(y_{3}\right)\right] \\
& =\sum_{\emptyset \neq I \subseteq[3], 1 \notin I} \lambda^{|I|-1}\left[P\left(y_{1}\right), \hat{P}_{I}\left(y_{2}\right), \hat{P}_{I}\left(y_{3}\right)\right]+\sum_{\emptyset \neq I \subseteq[3], 1 \in I} \lambda^{[I]-1}\left[y_{1}, \hat{P}_{I}\left(y_{2}\right), \hat{P}_{I}\left(y_{3}\right)\right] \\
& =\sum_{\emptyset \neq I \subseteq[3], 1 \notin I I} \lambda^{|I|-1}\left[\left[P\left(x_{1}\right), P\left(x_{2}\right), P\left(x_{3}\right)\right], \hat{P}_{I}\left(y_{2}\right), \hat{P}_{I}\left(y_{3}\right)\right]
\end{aligned}
$$




$$
\begin{aligned}
& +\sum_{\emptyset \neq I \subseteq[3], 1 \in I} \lambda^{|I|-1}\left[\sum_{\emptyset \neq J \subseteq[3]} \lambda^{|J|-1}\left[\hat{P}_{J}\left(x_{1}\right), \hat{P}_{J}\left(x_{2}\right), \hat{P}_{J}\left(x_{3}\right)\right], \hat{P}_{I}\left(y_{2}\right), \hat{P}_{I}\left(y_{3}\right)\right] \\
= & \sum_{\emptyset \neq K \subseteq[5], K \cap[3]=\emptyset} \lambda^{|K|-1}\left[\left[\hat{P}_{K}\left(x_{1}\right), \hat{P}_{K}\left(x_{2}\right), \hat{P}_{K}\left(x_{3}\right)\right], \hat{P}_{K}\left(x_{4}\right), \hat{P}_{K}\left(x_{5}\right)\right] \\
& +\sum_{\emptyset \neq I \subseteq[5], I \cap[3] \neq \emptyset} \lambda^{|I|-1}\left[\left[\hat{P}_{K}\left(x_{1}\right), \hat{P}_{K}\left(x_{2}\right), \hat{P}_{K}\left(x_{3}\right)\right], \hat{P}_{K}\left(x_{4}\right), \hat{P}_{K}\left(x_{5}\right)\right] \\
= & \sum_{\emptyset \neq I \subseteq[5]} \lambda^{|I|-1}\left[\left[\hat{P}_{K}\left(x_{1}\right), \hat{P}_{K}\left(x_{2}\right), \hat{P}_{K}\left(x_{3}\right)\right], \hat{P}_{K}\left(x_{4}\right), \hat{P}_{K}\left(x_{5}\right)\right] .
\end{aligned}
$$

Since $(L,[,]$,$) is a 3-Lie algebra, for any given \emptyset \neq I \subseteq[5]$, we have

$$
\begin{aligned}
& {\left[\left[\hat{P}_{K}\left(x_{1}\right), \hat{P}_{K}\left(x_{2}\right), \hat{P}_{K}\left(x_{3}\right)\right], \hat{P}_{K}\left(x_{4}\right), \hat{P}_{K}\left(x_{5}\right)\right]=\left[\left[\hat{P}_{K}\left(x_{1}\right), \hat{P}_{K}\left(x_{4}\right), \hat{P}_{K}\left(x_{5}\right)\right], \hat{P}_{K}\left(x_{2}\right), \hat{P}_{K}\left(x_{3}\right)\right]} \\
& \quad+\left[\left[\hat{P}_{K}\left(x_{2}\right), \hat{P}_{K}\left(x_{4}\right), \hat{P}_{K}\left(x_{5}\right)\right], \hat{P}_{K}\left(x_{3}\right), \hat{P}_{K}\left(x_{1}\right)\right]+\left[\left[\hat{P}_{K}\left(x_{3}\right), \hat{P}_{K}\left(x_{4}\right), \hat{P}_{K}\left(x_{5}\right)\right], \hat{P}_{K}\left(x_{1}\right), \hat{P}_{K}\left(x_{2}\right)\right] .
\end{aligned}
$$

Thus from the above sum, we conclude that $\left(L,[,,]_{P}\right)$ is a 3-Lie algebra.

Further we have

$$
\begin{aligned}
{\left[P\left(x_{1}\right), P\left(x_{2}\right), P\left(x_{3}\right)\right]_{P} } & =\sum_{\emptyset \neq I \subseteq[3]} \lambda^{|I|-1}\left[\hat{P}_{I}\left(P\left(x_{1}\right)\right), \hat{P}_{I}\left(P\left(x_{2}\right)\right), \hat{P}_{I}\left(P\left(x_{3}\right)\right)\right] \\
& =\sum_{\emptyset \neq I \subseteq[3]} \lambda^{|I|-1}\left[P\left(\hat{P}_{I}\left(x_{1}\right)\right), P\left(\hat{P}_{I}\left(x_{2}\right)\right), P\left(\hat{P}_{I}\left(x_{3}\right)\right)\right] \\
& =\sum_{\emptyset \neq I \subseteq[3]} \lambda^{|I|-1} P\left(\left[\hat{P}_{I}\left(x_{1}\right), \hat{P}_{I}\left(x_{2}\right), \hat{P}_{I}\left(x_{3}\right)\right]_{P}\right) .
\end{aligned}
$$

This proves that $P$ is a Rota-Baxter operator on $\left(L,[,]_{P}\right)$.

Theorem 5.2. Let $(L,[,], P$,$) be a Rota-Baxter 3-Lie algebra of weight \lambda$. Let d be a differential operator of weight $\lambda$ on $L$ satisfying $d P=P d$. Then $d$ is a derivation of weight $\lambda$ on the 3-Lie algebra $\left(L,[,]_{P}\right)$, where $[,,]_{P}$ is defined in Eq. (30).

Proof. Let $x_{1}, x_{2}, x_{3} \in L$. Using the notation in Eqs. (7) and (9), we have

$$
\begin{aligned}
d\left(\left[x_{1}, x_{2}, x_{3}\right]_{P}\right) & =\sum_{\emptyset \neq I \subseteq[3]} \lambda^{|I|-1} d\left[\hat{P}_{I}\left(x_{1}\right), \hat{P}_{I}\left(x_{2}\right), \hat{P}_{I}\left(x_{3}\right)\right] \\
& =\sum_{\emptyset \neq I \subseteq[3]} \lambda^{|I|-1}\left(\sum_{\emptyset \neq J \subseteq[3]} \lambda^{|J|-1}\left[\check{d}_{J} \hat{P}_{I}\left(x_{1}\right), \check{d}_{J} \hat{P}_{I}\left(x_{2}\right), \check{d}_{J} \hat{P}\left(x_{3}\right)\right]\right) \\
& =\sum_{\emptyset \neq J \subseteq[3]} \lambda^{|J|-1}\left(\sum_{\emptyset \neq I \subseteq[3]} \lambda^{|I|-1}\left[\hat{P}_{I} \check{d}_{J}\left(x_{1}\right), \hat{P}_{I} \check{d}_{J}\left(x_{2}\right), \hat{P} \breve{d}_{J}\left(x_{3}\right)\right]\right) \\
& =\sum_{\emptyset \neq J \subseteq[3]} \lambda^{|J|-1}\left[\check{d}_{J}\left(x_{1}\right), \breve{d}_{J}\left(x_{2}\right), \check{d}_{J}\left(x_{3}\right)\right]_{P} .
\end{aligned}
$$

Therefore, $d$ is a derivation of weight $\lambda$ on the 3 -Lie algebra $\left(L,[,,]_{P}\right)$. 
Corollary 5.3. Let $(L,[,]$,$) be a 3-Lie algebra, d$ be a invertible derivation of $L$ of weight $\lambda$. Then $\left(L,[x, y, z]_{d^{-1}}\right)$ with $[,$,$] defined in Eq. (30) is a 3-Lie algebra. Further$

$$
[x, y, z]_{d^{-1}}=d\left(\left[d^{-1}(x), d^{-1}(y), d^{-1}(z)\right]\right) \text { for all } x, y, z \in L,
$$

and $d$ is a derivation of weight $\lambda$ on the 3 -Lie algebra $\left(L,[,,]_{d^{-1}}\right)$.

Proof. By Theorem 2.2, $d^{-1}$ is a Rota-Baxter operator of weight $\lambda$ on the 3-Lie algebra $(L,[,]$,$) .$ Then by Theorem 5.1, $d^{-1}$ is a Rota-Baxter operator on the 3-Lie algebra $L$ equipped with the multiplication $[x, y, z]_{d^{-1}}$ defined in Eq. (30). By Eq. (9) we have

$$
[x, y, z]_{d^{-1}}=d\left(d^{-1}\left([x, y, z]_{d^{-1}}\right)\right)=d\left[d^{-1}(x), d^{-1}(y), d^{-1}(z)\right],
$$

as needed. The last statement follows from Theorem 5.2.

Corollary 5.4. Let $(L,[], P$,$) be a Rota-Baxter Lie algebra of weight \lambda$ and let $f \in L^{*}$. Suppose $f$ and $P$ satisfy

$$
\left.f([x, y])=0, \quad\left(P+\lambda I d_{L}\right)(f(x)[P(y), P(z)]+f(y)[P(z), P(x)])+f(z)[P(x), P(y)]\right)=0 .
$$

Define

$$
\begin{aligned}
& {[x, y, z]_{f, P}:=f(P(x))([P(y), z]+[y, P(z)]+\lambda[y, z])+f(P(y))([P(z), x]+[z, P(x)]+\lambda[z, x])} \\
& \quad+f(P(z))([P(x), y]+[y, P(x)]+\lambda[x, y])+f(x)([P(y), P(z)]+\lambda[P(y), z]+\lambda[y, P(z)] \\
& \left.\quad+\lambda^{2}[y, z]\right)+f(y)\left([P(z), P(x)]+\lambda[P(z), x]+\lambda[z, P(x)]+\lambda^{2}[z, x]\right) \\
& \quad+f(z)\left([P(x), P(y)]+\lambda[P(x), y]+\lambda[x, P(y)]+\lambda^{2}[x, y]\right) \text { for all } x, y, z \in L .
\end{aligned}
$$

Then $\left(L,[,,]_{f, P}, P\right)$ is a Rota-Baxter 3-Lie algebra of weight $\lambda$.

Proof. By Theorem 3.2.

$$
[x, y, z]_{f}:=f(x)[y, z]+f(y)[z, x]+f(z)[x, y]
$$

defines a 3-Lie algebra on $L$ for which $P$ is a Rota-Baxter operator of weight $\lambda$. Then by Theorem 5.1, the derived ternary multiplication $[,,]_{f, P}$ from $[,]:,=[,,]_{f}$ defined in Eq. (30) also equips $L$ with a 3-Lie algebra structure for which $P$ is a Rota-Baxter operator of weight $\lambda$. By direct checking, we see that $[,,]_{f, P}$ thus obtained agrees with the one defined in Eq. (32).

Taking the case when $\lambda=0$, we obtain

Corollary 5.5. Let $(L,[], P$,$) be a Rota-Baxter Lie algebra of weight zero, and f \in L^{*}$. If $f$ and $P$ satisfy

$$
f([x, y])=0, P(f(x)[P(y), P(z)]+f(y)[P(z), P(x)])+f(z)[P(x), P(y)])=0,
$$

then $\left(L,[,]_{P}, P\right)$ is a a Rota-Baxter 3-Lie algebra of weight zero, where

$$
\begin{aligned}
{[x, y, z]_{P}=} & f(P(x))([P(y), z]+[y, P(z)])+f(P(y))([P(z), x]+[z, P(x)]) \\
& +f(P(z))([P(x), y]+[y, P(x)])+f(x)[P(y), P(z)] \\
& +f(y)[P(z), P(x)])+f(z)[P(x), P(y)] \text { for all } x, y, z \in L .
\end{aligned}
$$


Remark 5.6. For a Rota-Baxter 3-Lie algebra $(L,[,], P$,$) of weight zero, L may not be a 3-Lie$ algebra with the multiplication

$$
[x, y, z]_{1}=[P(x), y, z]+[x, P(y), z]+[x, y, P(z)] \text { for all } x, y, z \in L .
$$

For Example, let L be a 3-Lie algebra in the multiplication

$$
\left[x_{1}, x_{2}, x_{3}\right]=x_{4},\left[x_{1}, x_{2}, x_{4}\right]=x_{3},\left[x_{1}, x_{3}, x_{4}\right]=x_{2},\left[x_{2}, x_{3}, x_{4}\right]=x_{1} \text {, }
$$

where $\left\{x_{1}, x_{2}, x_{3}, x_{4}\right\}$ is a basis of $L$. Since $D=\operatorname{ad}\left(x_{1}, x_{2}\right)+\operatorname{ad}\left(x_{3}, x_{4}\right)$ is an invertible derivation of $L$ and $D^{-1}=D, D$ is a Rota-Baxter of weight zero on $(L,[,]$,$) . And$

$$
\begin{aligned}
& {\left[x_{1}, x_{2}, x_{3}\right]_{1}=\left[D\left(x_{1}\right), x_{2}, x_{3}\right]+\left[x_{1}, D\left(x_{2}\right), x_{3}\right]+\left[x_{1}, x_{2}, D\left(x_{3}\right)\right]=x_{3},} \\
& {\left[x_{1}, x_{2}, x_{4}\right]_{1}=\left[D\left(x_{1}\right), x_{2}, x_{4}\right]+\left[x_{1}, D\left(x_{2}\right), x_{4}\right]+\left[x_{1}, x_{2}, D\left(x_{4}\right)\right]=x_{4},} \\
& {\left[x_{1}, x_{3}, x_{4}\right]_{1}=\left[D\left(x_{1}\right), x_{3}, x_{4}\right]+\left[x_{1}, D\left(x_{3}\right), x_{4}\right]+\left[x_{1}, x_{3}, D\left(x_{4}\right)\right]=x_{1},} \\
& {\left[x_{2}, x_{3}, x_{4}\right]_{1}=\left[D\left(x_{2}\right), x_{3}, x_{4}\right]+\left[x_{2}, D\left(x_{3}\right), x_{4}\right]+\left[x_{2}, x_{3}, D\left(x_{4}\right)\right]=x_{2} .}
\end{aligned}
$$

Since $\left[\left[x_{1}, x_{2}, x_{3}\right]_{1}, x_{2}, x_{4}\right]_{1}=-x_{2}$, and

$$
\left[\left[x_{1}, x_{2}, x_{4}\right]_{1}, x_{2}, x_{3}\right]_{1}+\left[x_{1},\left[x_{2}, x_{2}, x_{4}\right]_{1}, x_{3}\right]_{1}+\left[x_{1}, x_{2},\left[x_{3}, x_{2}, x_{4}\right]_{1}\right]_{1}=x_{2},
$$

L is not a 3-Lie algebra in the multiplication $[,,]_{1}$.

5.2. Rota-Baxter Lie triple systems. A Lie triple system [B]] is a vector space $L$ equipped with a ternary linear bracket $\{,\}:, L \otimes L \otimes L \rightarrow L$, satisfying

$$
\begin{gathered}
\{x, y, y\}=0, \\
\{x, y, z\}+\{y, z, x\}+\{z, x, y\}=0, \\
\{\{x, y, z\}, a, b\}=\{\{x, a, b\}, y, z\}+\{x,\{y, a, b\}, z\}+\{x, y,\{z, a, b\}\} \text { for all } x, y, z \in L .
\end{gathered}
$$

It is important in the study of symmetric spaces.

A Lie triple system is a 3-Lie algebra and thus it makes sense to define a Rota-Baxter Lie triple system.

Theorem 5.7. Let $(L,[,], P$,$) be a Rota-Baxter Lie triple system of weight \lambda$. Define a ternary multiplication on $L$ by $[,,]_{P}: L \otimes L \otimes L \rightarrow L$ in $E q .(30)$. Then $\left(L,[,,]_{P}, P\right)$ is a Rota-Baxter Lie triple system.

Proof. It is clear that $[x, y, y]_{P}=0$ and

$$
[x, y, z]_{P}+[y, z, x]_{P}+[z, x, y]_{P}=0 \text { for all } x, y, z \in L .
$$

Then the theorem follows from Theorem 5.1.

If $(L,[]$,$) is a Lie algebra, then (L,[,]$,$) is a Lie triple system [37], where [,$,$] is defined by$

$$
[x, y, z]:=[x,[y, z]] \text { for all } x, y, z \in L \text {. }
$$

Theorem 5.8. Let $(L,[], P$,$) be a Rota-Baxter Lie algebra of weight \lambda$. Then $(L,[,], P$,$) is a$ Rota-Baxter Lie triple system of weight $\lambda$. 
Proof. By Eqs. (35) and (10), we have

$$
\begin{aligned}
& {[P(x),[P(y), P(z)]]=[P(x), P[y, P(z)]]+[P(x), P[P(y), z]]+\lambda[P(x), P[y, z]] } \\
&=\quad P[P(x),[y, P(z)]]+P[x, P[y, P(z)]]+\lambda P[x,[y, P(z)]] \\
&+P[P(x),[P(y), z]]+P[x, P[P(y), z]]+\lambda P[x,[P(y), z]] \\
&+\lambda P[P(x),[y, z]]+\lambda P[x, P[y, z]]+\lambda^{2} P[x,[y, z]] .
\end{aligned}
$$

Since $P[x,[P(y), P(z)]]=P[x, P[y, P(z)]]+P[x, P[P(y), z]]+\lambda P[x, P[y, z]]$, we have

$$
\begin{aligned}
{[P(x), P(y), P(z)]=} & {[P(x),[P(y), P(z)]] } \\
= & P[P(x),[P(y), z]]+P[P(x),[y, P(z)]]+P[x,[P(y), P(z)]] \\
& +\lambda P[P(x),[y, z]]+\lambda P[x,[P(y), z]]+\lambda P[x,[y, P(z)]]+\lambda^{2} P[x,[y, z]] \\
= & P[P(x), P(y), z]+P[P(x), y, P(z)]+P[x, P(y), P(z)] \\
& +\lambda P[P(x), y, z]+\lambda P[x, P(y), z]+\lambda P[x, y, P(z)]+\lambda^{2} P[x, y, z]
\end{aligned}
$$

for all $x, y, z \in L$. Hence $P$ is a Rota-Baxter operator on the 3 -Lie triple $\operatorname{system}(L,[,]$,$) .$

Acknowledgements. Ruipu Bai was supported in part by the Natural Science Foundation of Hebei Province (A2010000194). Li Guo acknowledges support from NSF grant DMS 1001855.

\section{REFERENCES}

[1] M. Aguiar, Pre-Poisson algebras, Lett. Math. Phys. 54 (2000) 263-277. 1]

[2] H. An, C. Bai, From Rota-Baxter algebras to pre-Lie algebras. arXiv:0711-1389v1[math-ph]. 2

[3] J. A. de Azcarraga, J. M. Izquierdo, n-ary algebras: a review with applications, J. Phys. A: Math. Theor. 43 (2010) 293001, arXiv: 1005.1028 [math-ph].1]

[4] J. Bagger, N. Lambert, Gauge symmetry and supersymmetry of multiple M2-branes, Phys. Pev. D 77 (2008) 065008.1

[5] C. Bai, A unified algebraic approach to classical Yang-Baxter equation, J. Phys. A 40 (2007), 1107311082.2, 10

[6] C. Bai, O. Bellier, L. Guo and X. Ni, Spliting of operations, Manin products and Rota-Baxter operators, IMRN (2012). DOI: 10.1093/imrn/rnr266.1

[7] C. Bai, L. Guo and X. Ni, Generalizations of the classical Yang-Baxter equation and O-operators, $J$. Math. Phys. 52 (2011) 063515. 2, 10

[8] C. Bai, L. Guo and X. Ni, Nonabelian generalized Lax pairs, the classical Yang-Baxter equation and PostLie algebras, Comm. Math. Phys. 297 (2010) 553-596. 2

[9] R. Bai, C. Bai and J. Wang, Pealizations of 3-Lie algebras, J. Math. Phys., 51, 063505 (2010) 1, 2, 5

[10] R. Bai, W. Han and C. Bai, The generating index of an n-Lie algebra, J. Phys. A: Math. Theor. 44 (2011) 185201 (14pp). 1

[11] R. Bai , C. Shen and Y. Zhang, 3-Lie algebras with an ideal N, Linear Alg. Appl. 431 (2009) 673-700.1

[12] R. Bai, G. Song and Y. Zhang, On classification of n-Lie algebras, Front. Math. China, 2011, 6(4): 581-606. 11

[13] G. Baxter, An analytic problem whose solution follows from a simple algebraic identity, Pacific J. Math. 10 (1960), 731-742. 1

[14] P. Cartier, On the structure of free Baxter algebras, Adv. Math. 9 (1972), 253-265.1

[15] A. Connes, D. Kreimer, Hopf algebras, Renormalisation and Noncommutative Geometry, Comm. Math. Phys.199 (1988) 203-242 1]

[16] A.S. Dzhumadil' daev, Identities and derivations for Jacobi algebras, arXiv: math.RA/0202040v1.9

[17] K. Ebrahimi-Fard, L. Guo and D. Kreimer, Spitzer's identity and the algebraic Birkhoff decomposition in pQFT, J. Phys. A: Math. Gen. 37 (2004), 11037-11052. [1]

[18] K. Ebrahimi-Fard, L. Guo and D. Manchon, Birkhoff type decompositions and the Baker-CampbellHausdorff recursion, Comm. Math. Phys. 267 (2006) 821-845. 1 
[19] V.T. Filippov, $n$-Lie algebras, Sib. Mat. Zh., 26 (1985) 126-140. 1, 10

[20] L. Guo, WHAT IS a Rota-Baxter algebra, Notice Amer. Math. Soc. 56 (2009) 1436-1437. 1

[21] L. Guo, Introduction to Rota-Baxter Algebra, International Press and Higher Education Press, 2012. 11

[22] L. Guo and W. Keigher, Baxter algebras and shuffle products, Adv. Math., 150 (2000), 117-149.1]

[23] L. Guo and W. Keigher, On differential Rota-Baxter algebras, J. Pure Appl. Algebra, 212 (2008), 522540. 目

[24] L. Guo, W. Sit and R. Zhang, Differemtail type operators and Gröbner-Shirshov bases, J. Symolic Comput. (2012). 1

[25] L. Guo and B. Zhang, Renormalization of multiple zeta values, J. Algebra, 319 (2008), 3770-3809. 1, 2

[26] A. Gustavsson, Algebraic structures on parallel M2-branes, arXiv: 0709.1260. 1]

[27] P. Ho, M. Chebotar and W. Ke, On skew-symmetric maps on Lie algebras, Proc. Royal Soc. Edinburgh A 113 (2003) 1273-1281. 1

[28] P. Ho, R. Hou and Y. Matsuo, Lie 3-algebra and multiple $M_{2}$-branes, arXiv: 0804.2110.1]

[29] P. Ho, Y. Imamura, Y. Matsuo, M2 to D2 revisited, JHEP, 0807:003, 2008. DOI: 10.1088/11266708/2008/07/003 1

[30] N. Jacobson, Lie and Jordan triple systems Amer. J. Math. 71 (1949) 149C170. 13

[31] X.X. Li, D.P. Hou and C.M. Bai, Rota-Baxter operators on pre-Lie algebras, J. Nonlinear Math. Phy., 14 (2007), no. 2, 269-289. 2, 6

[32] D. Manchon and S. Paycha, Nested sums of symbols and renormalised multiple zeta values, Int. Math. Res. Papers 2010 issue 24, 4628-4697 (2010). 2

[33] Y. Nambu, Generalized Hamiltonian dynamics, Phys. Pev. D 7 (1973) 2405-2412.1]

[34] G. Papadopoulos, M2-branes, 3-Lie algebras and Plucker relations, arXiv: 0804.2662.1]

[35] S. Kasymov, On a theory of $n$-Lie algebras, Algebra i Logika 26 (1987) 277-297. 1

[36] W. Ling, On the structure of $n$-Lie algebras, Dissertation, University-GHS-Siegen, Siegn, 1993. 11

[37] W.G. Lister, A structure theory of Lie triple systems, Trans. Amer. Math. Soc. 72 (1952) 217C242 13

[38] G.-C. Rota, Baxter algebras and combinatorial identities I, II, Bull. Amer. Math. Soc. 75 (1969), 325329, 330-334. 11

[39] G.-C. Rota, Baxter operators, an introduction, In: "Gian-Carlo Rota on Combinatorics, Introductory papers and commentaries”, Joseph P. S. Kung, Editor, Birkhäuser, Boston, 1995. 1

[40] M. A. Semenov-Tian-Shansky, What is a classical r-matrix?, Funct. Ana. Appl., 17 (1983) 259-272. 2, 10

[41] L. Takhtajan, On foundation of the generalized Nambu mechanics, Comm. Math. Phys. 160 (1994) 295315. 1

College of Mathematics and Computer Science, Hebei University, Baoding 071002, China

E-mail address: bairp1@yahoo.com.cn

Department of Mathematics and Computer Science, Rutgers University, Newark, NJ 07102, USA

E-mail address: liguo@rutgers . edu

College of Mathematics and Computer Science, Hebei University, Baoding 071002, China

E-mail address: lijiaqianjiayou@163.com

College of Mathematics and Computer Science, Hebei University, Baoding 071002, China

E-mail address: wuyg1022@sina.com 